\title{
Compatible and Incompatible Interactions in Wheat Involving the Bt-10 Gene for Resistance to Tilletia tritici, the Common Bunt Pathogen
}

\author{
Denis A. Gaudet, Zhen-Xiang Lu, Frances Leggett, Bryan Puchalski, and André Laroche
}

Agriculture and Agri-Food Canada, Lethbridge Research Centre, P.O. Box 3000, Lethbridge, Alberta, Canada T1J 4B1. Accepted for publication 1 May 2007.

\begin{abstract}
Gaudet, D. A., Lu, Z.-X., Leggett, F., Puchalski, B., and Laroche, A. 2007. Compatible and incompatible interactions in wheat involving the Bt-10 gene for resistance to Tilletia tritici, the common bunt pathogen. Phytopathology 97:1397-1405.

The infection of wheat lines Neepawa (susceptible), and its sib BW553 that is nearly isogenic for the Bt-10 resistance gene by differentially virulent races T1 and T27 of common bunt (Tilletia tritici), was followed for 21 days following seeding (dfs) using fluorescence and confocal microscopy. Spore germination was nonsynchronous and all spore stages including germination were observed 5 to $21 \mathrm{dfs}$. Initial host perception of pathogen invasion, based on autofluorescence in epidermal cells adjacent to the appressoria, was similar in both compatible and incompatible interactions, and occurred as early as 5 to $6 \mathrm{dfs}$. The total number of sites on a $1-\mathrm{cm}$ segment of coleoptile adjacent to the seed that ex-

as late as $21 \mathrm{dfs}$. In the compatible interaction, the autofluorescence became more diffuse 10 to $12 \mathrm{dfs}$, emanating in all directions in association with fungal spread. In the incompatible interaction, autofluorescence remained restricted to a small area surrounding the penetration site. Two different reaction zones that extended further in tissues surrounding the penetration point in the incompatible interaction compared with the compatible interaction were identified. The accumulation of callose around invading fungal hyphae was observed during both the compatible and incompatible interactions from 8 to $21 \mathrm{dfs}$. While callose accumulation was more extensive and widespread in the incompatible interaction, it was clearly present in compatible interactions, particularly in treatments involving BW553. These results were confirmed by expression of callose synthase transcripts that were more abundant in BW553 than in Neepawa and were upregulated during pathogen infection in both compatible and incompatible interactions.
\end{abstract} hibited autofluorescence was similar in both the compatible and incompatible interactions and rose to a maximum of 35 to 40 per $1 \mathrm{~cm}$ length of coleoptile following $17 \mathrm{dfs}$, although new infection events were observed
Additional keywords: defense responses, disease resistance, gene expression.
Plant disease resistance responses can be divided into a series of interrelated stages $(3,20,21,38)$. There is an initial recognition of the pathogen by the host plant that frequently involves the interaction between resistance-genes and pathogen avirulence genes encoding specific effectors or elicitors. Usually, an incompatible interaction results in increases in endogenous levels of reactive oxygen intermediates, nitric oxide, salicylic acid (SA), jasmonic acid (JA), and ethylene that trigger the defense responses through different signaling pathways. Signaling pathways activate a series of defense responses that curb or eliminate the pathogen. These responses include the hypersensitive response (HR), upregulation of phenylalanine ammonium lyase, a key enzyme in plant defense, and synthesis of a wide range of antimicrobial compounds including pathogenesis related (PR)-proteins and phytoalexins (38). Additionally, host plants can reinforce cell walls by depositing cell wall reinforcing materials such as lignin and callose (27). Callose, a (1-3)- $\beta$-D-glucan, frequently accumulates in response to mechanical damage or patho-

Corresponding author: D. A. Gaudet; E-mail address: gaudetd@agr.gc.ca

* The $e$-Xtra logo stands for "electronic extra" and indicates that the online version contains supplemental material not included in the print edition. The supplemental figure shows the progressive layering of callose around the invading hypha in the coleoptile of Taber wheat in the incompatible interaction involving Tilletia tritici race $\mathrm{T} 1$ and $B t-10$ in Taber wheat at 13 days following seeding.

doi:10.1094/PHYTO-97-11-1397

This article is in the public domain and not copyrightable. It may be freely reprinted with customary crediting of the source. The American Phytopathological Society, 2007 gen infection and may provide a physical barrier to penetration by pathogens (23). However, contradictory roles for callose in plant defense response have been proposed and its contribution remains to be established $(27,28)$.

Common bunt, incited by the fungi Tilletia tritici (Bjerk.) Wint and T. laevis Kühn, can be a serious disease, causing yield and quality losses in spring and winter wheat (Triticum aestivum L.) $(1,15)$. These pathogens are obligate biotrophs and a gene-forgene interaction exists between avirulence genes of the bunt pathogen and the many individual host resistance Bt-genes (10, 16). The Bt-10 gene is one of approximately 15 known Bt-genes that is effective against the majority of common bunt races and is an important resistance source for new wheat varieties in many parts of the world $(6,8,16)$.

Germination of the bunt fungus teliospore coincides with germination of the wheat seedling shortly after seeding; spore germination generally occurs 4 to 10 days after seeding $(24,31)$ and penetration of emerging wheat coleoptiles occurs 7 to 10 days postinoculation $(34,39)$. Secondary binucleate and multinucleate hyphae, resulting from the fusion between two haploid sporidia, may produce secondary multinucleate sporidia (11) or may directly penetrate the emerging coleoptile $(34,39)$ and spread both inter- or intracellularly (39). Penetration is preceded by the formation of thick walled, irregularly shaped, and deeply staining infection mycelia, from which an appressorium is formed and penetration occurs (2). In a compatible interaction, a successful infection results when the fungus is able to spread inwards from the coleoptile to establish itself in the region directly below the apical growing point of the developing culm following intercellu- 
lar passage through several embryonic leaves, a process which takes 3 to 5 weeks after seeding $(5,34)$, although macroscopic symptoms (i.e., presence of the infected heads) are only visible near plant maturity (15).

Compatible and incompatible reactions to common bunt in wheat that involve resistance expression have been described $(2,14,39)$. Infection processes were identical in both compatible and incompatible interactions 6 days following seeding (dfs) and growth at $15^{\circ} \mathrm{C}$, but fungal growth remained restricted to the coleoptile after 9 days in the incompatible interaction, whereas in the compatible interaction, the bunt fungus had frequently grown through the coleoptile and invaded the first embryonic leaf (39). Additionally in the incompatible interaction, a thickening in the epidermal layer in a zone just below the site of penetration or a gelatinization between the plasma membrane and the cuticle shortly following penetration was observed $(14,39)$. A similar rapid deposition of interfacial matrix surrounding the invading fungal hyphae has been reported in the incompatible interaction with barley and the covered smut pathogen, Ustilago hordei (Pers.) Lagerh., shortly following penetration (17). In both instances, upon entry of the hypha into the intercellular space between cells, a conical sheath composed of the gelatin-like substance that enveloped the invading hyphae was associated with termination of fungal growth $(14,17,39)$. No evidence of an HR has been observed in wheat infected by bunt $(2,14,39)$, but a localized necrosis involving two to three cells was observed in the incompatible interaction involving $U$. hordei, 2 days following inoculation (17).

In a previous study, we characterized defense-related genes that were preferentially upregulated during an incompatible interaction involving the bunt resistance gene Bt-10 (25). Several genes including a $P R-1.1$ were differentially upregulated in the incompatible interaction between the Bt-10 gene for common bunt resistance and an avirulent race of $T$. tritici compared to a com- patible interaction, as early as 10 to $15 \mathrm{dfs}$ (25). In the present study, we employed fluorescent and confocal microscopy to compare host responses for 20 days in compatible and incompatible interactions involving the common bunt fungus $T$. tritici and the $B t-10$ gene.

\section{MATERIALS AND METHODS}

Biological materials and growth conditions. The following spring wheat (Triticum aestivum L. em. Thell) cultivars and lines were employed: line BW553 (pedigree = Neepawa*6//Red Bobs/PI 178383), a near isogenic sib of the cv. Neepawa, and Taber (pedigree $=\mathrm{HY} 320 * 2 / \mathrm{BW} 553$ ) as the $B t-10$ resistant cultivars, and Neepawa and HY320 as susceptible cultivars $(6,8)$. The $T$. tritici races $\mathrm{T} 1$ and $\mathrm{T} 27$ are virulent on Neepawa and HY320, whereas T1 is avirulent on $B t-10$ while $\mathrm{T} 27$ is virulent on Bt-10 $(7,8,16)$.

All seeds were surface sterilized in $10 \%$ bleach plus Tween 20 $(0.1 \%, \mathrm{vol} / \mathrm{vol})$ for $3 \mathrm{~min}$, rinsed three times in sterile distilled water, dried, and stored at $4^{\circ} \mathrm{C}$ until used. T. tritici teliospores were thoroughly dusted on the wheat seeds just before seeding. Seeds were planted into rootrainers (Spencer-Lemaire Industries, Edmonton, AB, Canada) containing a soilless potting mixture, with the germ end down. This ensured formation of a straight coleoptile that always elongated in parallel with the seed and was important to subsequent histological studies. Rootrainers were placed in a growth cabinet (Conviron) set at $12^{\circ} \mathrm{C}\left( \pm 1.5^{\circ} \mathrm{C}\right)$, the optimum temperature for infection for common bunt (7). Fluorescent and incandescent lights $\left(\approx 240 \mu \mathrm{mol} \mathrm{s}^{-1} \mathrm{~m}^{-2}\right)$ in growth cabinets and greenhouses provided an $18 \mathrm{~h}$ day-length.

Microscopic studies. For microscopic studies, plants were sampled at daily intervals from 4 to $21 \mathrm{dfs}$. Plants were gently washed and crown tissues consisting of a $1-\mathrm{cm}$ section of the

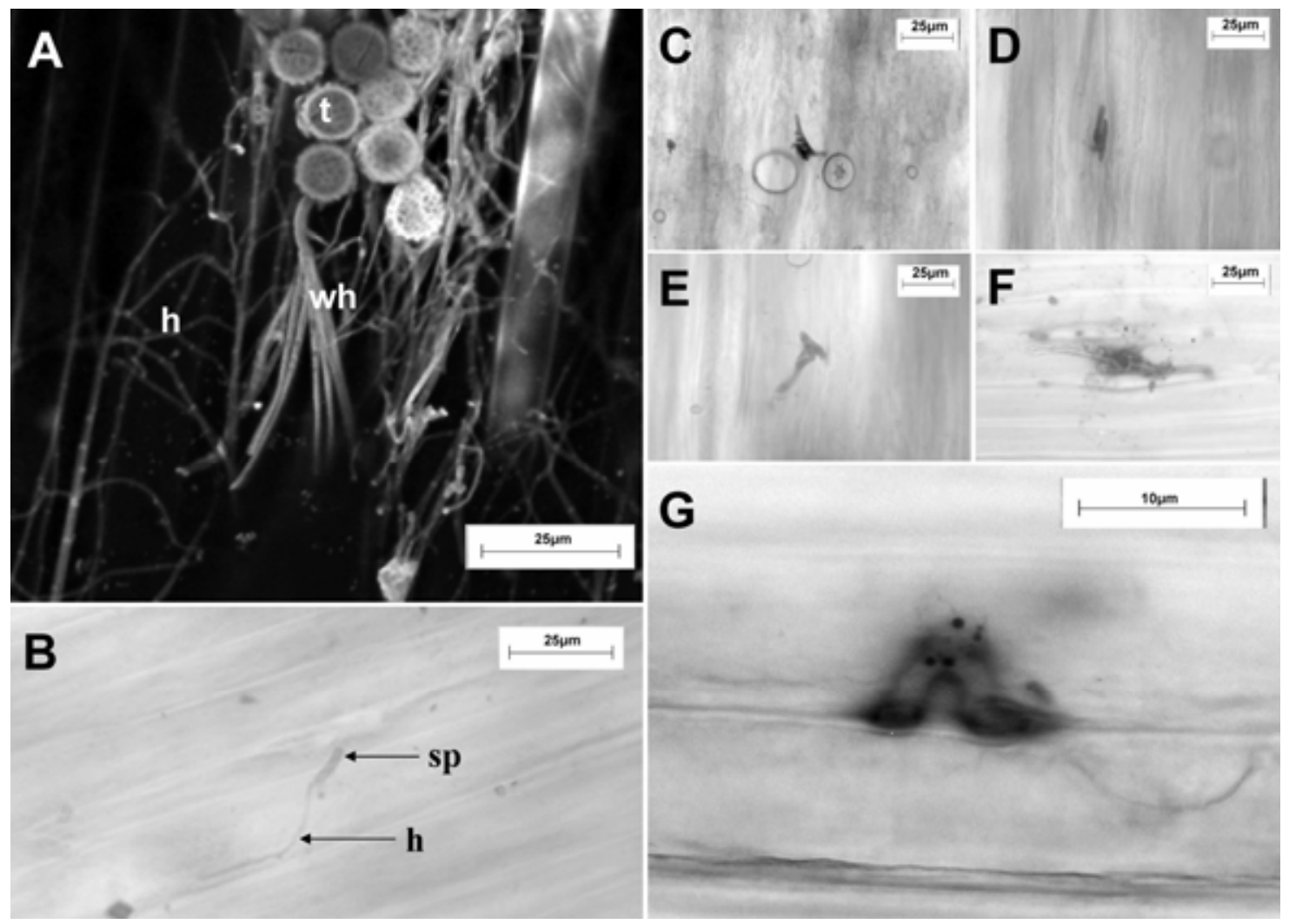

Fig. 1. Tilletia tritici germination and development of the surface of wheat coleoptiles. A, Confocal, and $\mathbf{B}$ to G, brightfield microscopic, stained with lactophenolcotton blue. A, Race T1 teliospores (t) germinating to produce whorl of primary sporidia (wh) and extension of primary hyphae (h) over the surface of the coleoptile in Neepawa, 12 days following seeding (dfs); B, secondary sporidia (sp) and developing hyphae (h) of race T1 on HY320, aligning itself along the longitudinal axis of the intercellular groove; C to G, knobby, contorted appressoria formed prior to penetrating the coleoptile; C and D, race T1 on BW553, 6 dfs; $\mathbf{E}$, race T27 on BW553, 6 dfs; $\mathbf{F}$, race T1 on BW553,13 dfs; and $\mathbf{G}$, race T27 on BW553, 13 dfs. 
coleoptile surrounding the crown region were excised, split longitudinally in half, and both halves were mounted in lactophenolcotton (aniline) blue (19). Sections were viewed with an Olympus BX80 research microscope using the 330 to $385 \mathrm{~nm}$ and 460 to $490 \mathrm{~nm}$ excitation and emission filters, respectively, and an HBO103W/2 light source. For confocal microscopy, the same preparation and stain procedure of lactophenol-cotton blue was employed except that sections were mounted in AquaMount (Polysciences, Inc., Warrington, PA) and dried overnight. The number of fluorescent infection sites on both halves of the $1-\mathrm{cm}$ coleoptile sections was counted from a minimum of five seedlings per replication. Three replications were employed and the study was repeated two times. Infection sites were verified by brightfield microscopy to ensure that fungal hyphae associated with the fluorescence were those of $T$. tritici and not contaminant fungi. For confocal microscopy, slides were viewed with a Zeiss Axiophot LSM 410 confocal microscope (Zeiss, Germany) using a $633 \mathrm{HeNe}$ laser. The material was optically z-sectioned and the resulting sequence of images was processed to form a 3D image. The sequence of images was used to build the figures using the $3 \mathrm{D}$ reconstruction program SURFdriver (3.5Beta6).

Gene expression studies. The time-course expression of callose synthase, in noninoculated, and in T1 and T27 inoculated Neepawa and BW553 plants, after 11 and 15 days (Zadoks stages 11 and 12, respectively, according to Tottman and Makepeace [36]) was determined by real-time polymerase chain reaction (PCR) analyses. Sequence data for wheat were identified from the GenBank database (National Center for Biotechnology Information, Bethesda, MD). The gene callose synthase (GenBank accession no. CA501322) originated from a wheat meiotic T. aestivum cDNA library. The sequences of the PCR primers, and the melting temperature $\left(\mathrm{T}_{\mathrm{m}}\right)$ are as follows: $\mathrm{F} 2$, tgaagacctcgaatctgcatt, $21 \mathrm{bp}$, $\mathrm{T}_{\mathrm{m}}=59.8^{\circ} \mathrm{C} ; \mathrm{R} 2$, tctggtttccttctcctttc, $21 \mathrm{bp}, \mathrm{T}_{\mathrm{m}}=59.7^{\circ} \mathrm{C}$. Primer pairs for real-time PCR analyses were designed using the software Primer3 (available online by Whitehead Institute and Howard Hughes Medical Institute). Guidelines for primer design were according to recommendations provided by QIAGEN (Mississauga, ON, Canada). Methods for RNA isolation and real time PCR were according to Lu et al. (25). Copy number presented for callose synthase reflects the numbers of transcripts per $50 \mathrm{ng}$ of total RNA.

\section{RESULTS}

The results of successful infection of seed inoculation with common bunt can only be evaluated at the end of the life cycle of the plant (i.e., in the grain head). Therefore, residual inoculated plants in each treatment and experiment were reared to maturity for determination of the percentage of bunted heads. Infection levels were consistently high in all experiments ranging from 60 to $70 \%$ in all susceptible (compatible) treatments. This indicated that environmental conditions were favorable for infection. Infection levels for incompatible interactions were $1.8 \%$, a level that is considered normal in a resistant reaction under the controlled environmental conditions employed $(7,8)$.

The first signs of teliospore germination were observed at $4 \mathrm{dfs}$ at $12^{\circ} \mathrm{C}$. All stages of germination of teliospores and subsequent fungal development reported by Woolman (39) and Churchward (2) including the formation of basidia, primary haploid sporidial whorls, H-bodies, secondary dikaryotic hyphae and sporidia, and infection hyphae were observed on the surface of the coleoptile (Fig. 1A to G). All of these spore germination stages were observed from 5 to $21 \mathrm{dfs}$ confirming the nonsimultaneous germination of the teliospores. Prior to the formation of infection structures, the secondary dikaryotic hyphae that originated from primary dikaryotic hyphae (Fig. 1A) or following germination of a dikaryotic secondary sporidia (Fig. 1B) became aligned longitudinally between two adjacent rows of epidermal cells of the coleoptile, as early as 5 to $6 \mathrm{dfs}$. Following this alignment, hyphae frequently became contorted, assuming a wide variety of shapes, some becoming compact and knobby (Figs. 1C to G, 2A and B, and $3 \mathrm{~B}$ to $\mathrm{D}$ ), some branching and proliferating to form small hyphal masses, (Fig. 1F) while others retained a normal, filamentous hyphal shape (Fig. 1G). These structures, referred to as appressoria (2), stained more intensely (Fig. 2A and B) with lactophenol-cotton blue compared with undifferentiated dikaryotic primary and secondary hyphae and sporidia (Fig. 1B).

The first visible sign of host reaction was an autofluorescence of cells in the vicinity of appressoria and was regularly observed on the surface of the coleoptile from 7 to $21 \mathrm{dfs}$ in inoculated treatments of both BW553 (Fig. 2A) and Neepawa (not shown). The autofluorescence first observed after 6 dfs, prior to penetration, became progressively more intense 1 and 2 days following formation of the appressorium (Fig. 2B), and remained visible in the coleoptile for $21 \mathrm{dfs}$ (Fig. 4). The early interactions observed from 6 to 8 dfs were identical in both BW553 and Neepawa (Fig. $2 \mathrm{~A}$ and $\mathrm{B}$; not shown for Neepawa). The numbers of fluorescing penetration and infection sites in the 1-cm section of the coleoptile were similar in both Neepawa and BW553 inoculated with race $\mathrm{T} 1$, rising to an average maximum number of 35 to 40 after 17 dfs (Fig. 4).

Clear evidence of enzymatic dissolution of the middle lamella between epidermal cells during penetration was observed; elevated, elongate openings were visible following penetration by

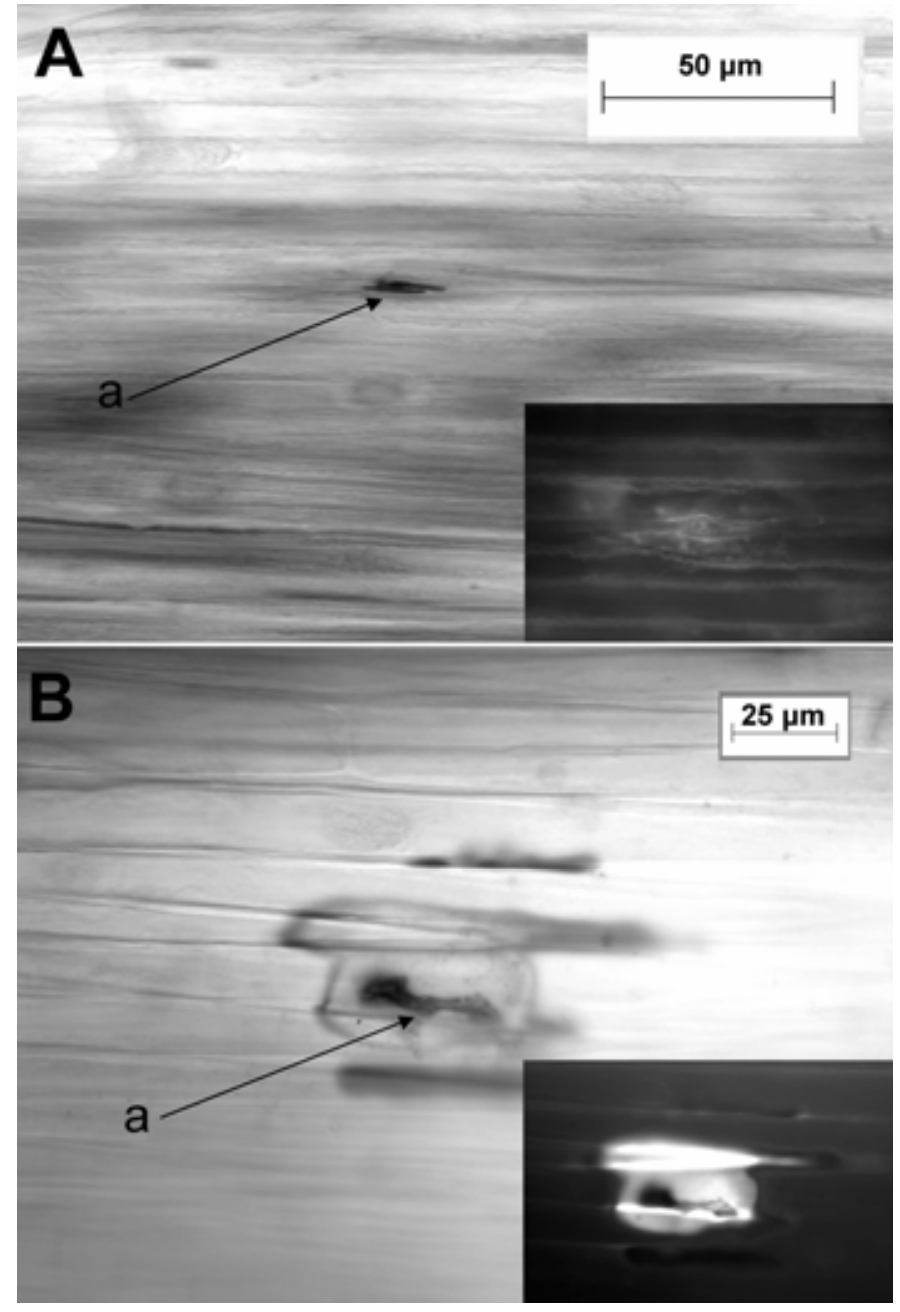

Fig. 2. Early infection events in wheat by Tilletia tritici race T1 in BW553. Brightfield and corresponding fluorescent images (inset). A, Slight autofluorescence in region surrounding an appressorium (a), 6 days following seeding (dfs); and $\mathbf{B}$, intense autofluorescence in region surrounding an appressorium $7 \mathrm{dfs}$. 
infecting hyphae (Figs. 1G and 5A). No differences in the formation of appressoria and the ensuing host reaction in terms of degree of autofluorescence, and in the early penetration events, were observed between incompatible and compatible interactions from 5 to $8 \mathrm{dfs}$. A region of the coleoptile surrounding the infection hyphae often became delineated following staining with lactophenol-cotton blue (Fig. 2B), and in some instances this stained region corresponded directly to the region of autofluorescence but with equal frequency, there was no delineation, or the delineation was not associated with autofluorescence.

Following 8 to $20 \mathrm{dfs}$, clear anatomical differences in the host reaction were observed between incompatible interactions involving T1 and BW553 and Taber, and compatible interactions involving T1 on Neepawa and HY320, and T27 on all cultivars. The earliest that microscopic droplets of callose appeared within cells adjacent to infection hyphae and coalescing around the infection hypha (Fig. 3A) was after 8 to $10 \mathrm{dfs}$. This corresponded to 4 to 6 days following from the first record of teliospore germination. In the earliest stages of callose accumulation in the incompatible reaction, production of callose originated in cells directly surrounding the infection site (Fig. 3A). Subsequently, in older infections, callose production was observed in cells as far as two cells away from those directly adjacent to the infection site (Fig. 3B and C). Eventually, most of the callose observed in the incompatible interaction appeared to become tightly appressed to the cell wall, the thickest accumulations were those closest to the infection hyphae (Fig. 3C and D). The deposition of callose around infection hyphae occurred in successive layers deposited over the hypha. Infection hyphae that became surrounded by callose appeared dark and lacked cytoplasm (Fig. 3D), and the cell wall appeared to lose its integrity (Fig. 5A). The infection site at $12 \mathrm{dfs}$ and corresponding digitized images illustrate that a complete encasement of the infection hyphae with callose had occurred (Fig. 5A and B).
Only small deposits of callose were observed in the compatible interaction, particularly in the early stages of infection around $10 \mathrm{dfs}$ (Figs. 5C and D and 6A to D). The extent of the autofluorescence associated with the infection site in these compatible interactions was similar in the early stages of penetration to that in the incompatible interaction between 8 to $10 \mathrm{dfs}$; however, the fluorescence subsequently expanded in all directions and became more diffuse. This corresponded to the intercellular spread of the fungus to deeper cell layers within the coleoptile 12 to $21 \mathrm{dfs}$ (Fig. 6C and D). Digital confocal images of compatible interaction of Neepawa inoculated with $\mathrm{T} 1$ revealed small callose deposits surrounding the hypha at the penetration site and scattered intercellularly at $12 \mathrm{dfs}$ (Fig. 5D). In the compatible interaction involving BW553 and race T27, larger, more pronounced callose

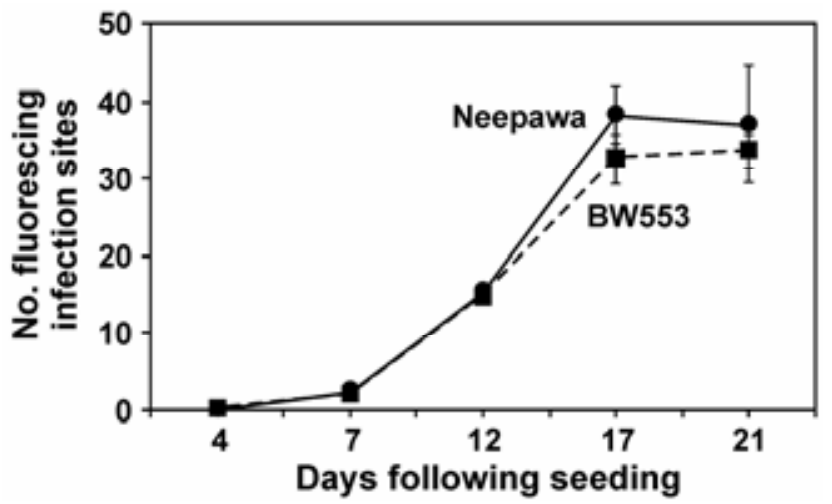

Fig. 4. Number of fluorescing infection sites observed on the surface of coleoptiles following seeding of susceptible Neepawa and resistant BW553 inoculated with race T1 of Tilletia tritici sampled 4 to 21 days following seeding. Bars indicate standard errors.
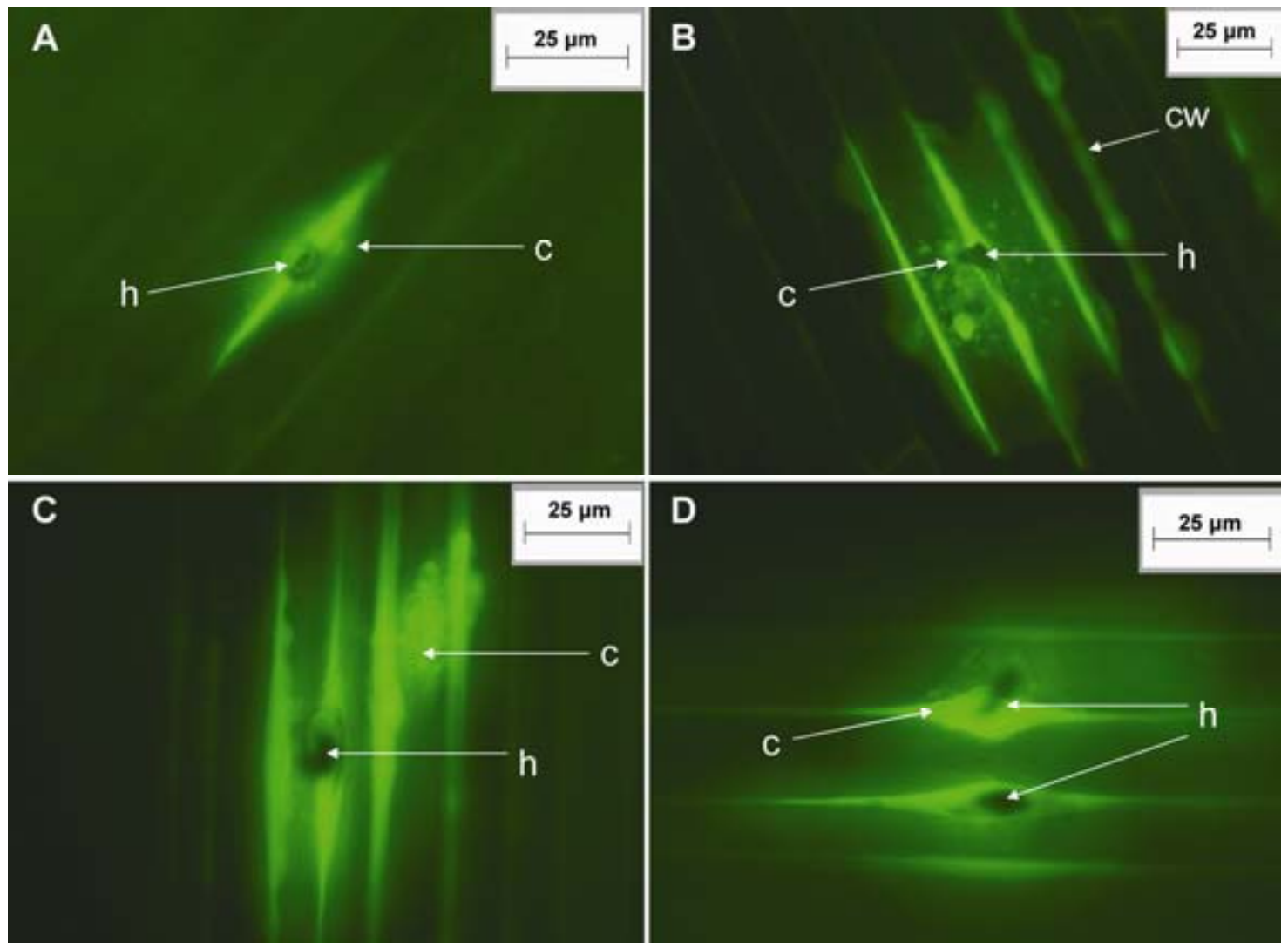

Fig. 3. Incompatible interaction involving Bt-10 in BW553 and race T1 of Tilletia tritici. A, Accumulation of callose (c) condensing around infection hypha (h) 8 days following seeding (dfs); $\mathbf{B}$ and $\mathbf{C}$, accumulation of callose in cells adjacent to cells directly associated with hyphal penetration 12 and 15 dfs, respectively. Callose accumulation along margins of cell walls (cw); and $\mathbf{D}$, two penetration sites showing differences in extent of callose deposition, $13 \mathrm{dfs}$. 
deposits were also observed at the penetration site and developed intercellularly to a greater extent as the fungus spread, compared with the compatible interactions involving Neepawa and races T1 or T27 (Fig. 7A and B versus 6A to D). The deposition of callose in the vicinity of the invading hyphae therefore appeared insufficient to block spread of the fungus to surrounding tissues in this compatible interaction.

Confocal imaging and digital analyses of the early stages (10 dfs) of the compatible and incompatible interactions revealed the presence of at least two different reaction zones, rz1 and rz2 (represented in red and blue, respectively, in Fig. 5B and D), in addition to the callose deposition directly around the invading hypha. In the incompatible interaction, the main reaction zone was $22 \mu \mathrm{M}$ in thickness (represented as rz1 in red in Fig. 5A) and extended well beyond the zone of callose accumulation as it encompassed the cells of the epidermis adjacent to the site of fungal penetration. In the compatible interaction, the corresponding reaction zone rz1 was globally much thinner $(4 \mu \mathrm{M}$ in Fig. 5D) than in the incompatible reaction and remained restricted to the surface of the external cells of the coleoptile, except for the region surrounding the infection hyphae that extended downward cylindrically corresponding to the downward extension of the fungus. Furthermore, in the compatible interaction there was no evidence of an enlargement of rz1 as hyphae spread intercellularly (Fig. 5C and D). A second zone, represented as $\mathrm{rz} 2$ in blue, appeared to be localized intercellularly between the two cells directly adjacent to the infection site. This zone was longer and extended downward to a greater extent in the incompatible compared with the compatible interaction (Fig. 5B and D).

Expression of callose synthase. Globally, the level of callose synthase transcripts was approximately two times higher in non- inoculated and inoculated BW553 treatments than in the corresponding Neepawa treatments, 11 and $15 \mathrm{dfs}$ (Fig. 8). The highest transcript levels were consistently observed in the T27-inoculated treatments (Fig. 8). Among the $11 \mathrm{dfs}$ treatments, transcript levels in T1- and T27-inoculated BW553 were 1.9 and 2.3 times higher $(P<0.05)$, respectively, than in the noninoculated control treatments. This trend, observed in the $15 \mathrm{dfs}$ treatments involving the T27-inoculated BW553 treatment but not in the T1-inoculated BW553 treatment, is likely a reflection of callose accumulation associated with the intercellular spread of T27. Additionally, these results demonstrate that the callose reaction in BW553 in the compatible interaction was more pronounced than in the compatible interaction with Neepawa, its near-isogenic sib.

\section{DISCUSSION}

We demonstrated a close association between penetration by the bunt pathogen and autofluorescence, in both the compatible interaction involving races $\mathrm{T} 1$ and $\mathrm{T} 27$ of $T$. tritici and the incompatible interaction with race $\mathrm{T} 1$ and the $B t-10$ resistance gene; this feature was employed to quantify penetration in the coleoptile. Incompatible interactions were distinguishable from compatible interacts only in the later stages of penetration with standard autofluorescence. Confocal microscopy and subsequent digital analyses permitted identification of two types of reaction zones that delineated compatible and incompatible interactions. The rapid accumulation of callose coalescing around the penetrating infection hyphae is the dominant microscopic feature of the incompatible interaction. However, callose is unlikely involved in a fungistatic or fungitoxic effect on the pathogen in the incompatible interaction and PR-proteins, and other plant-produced
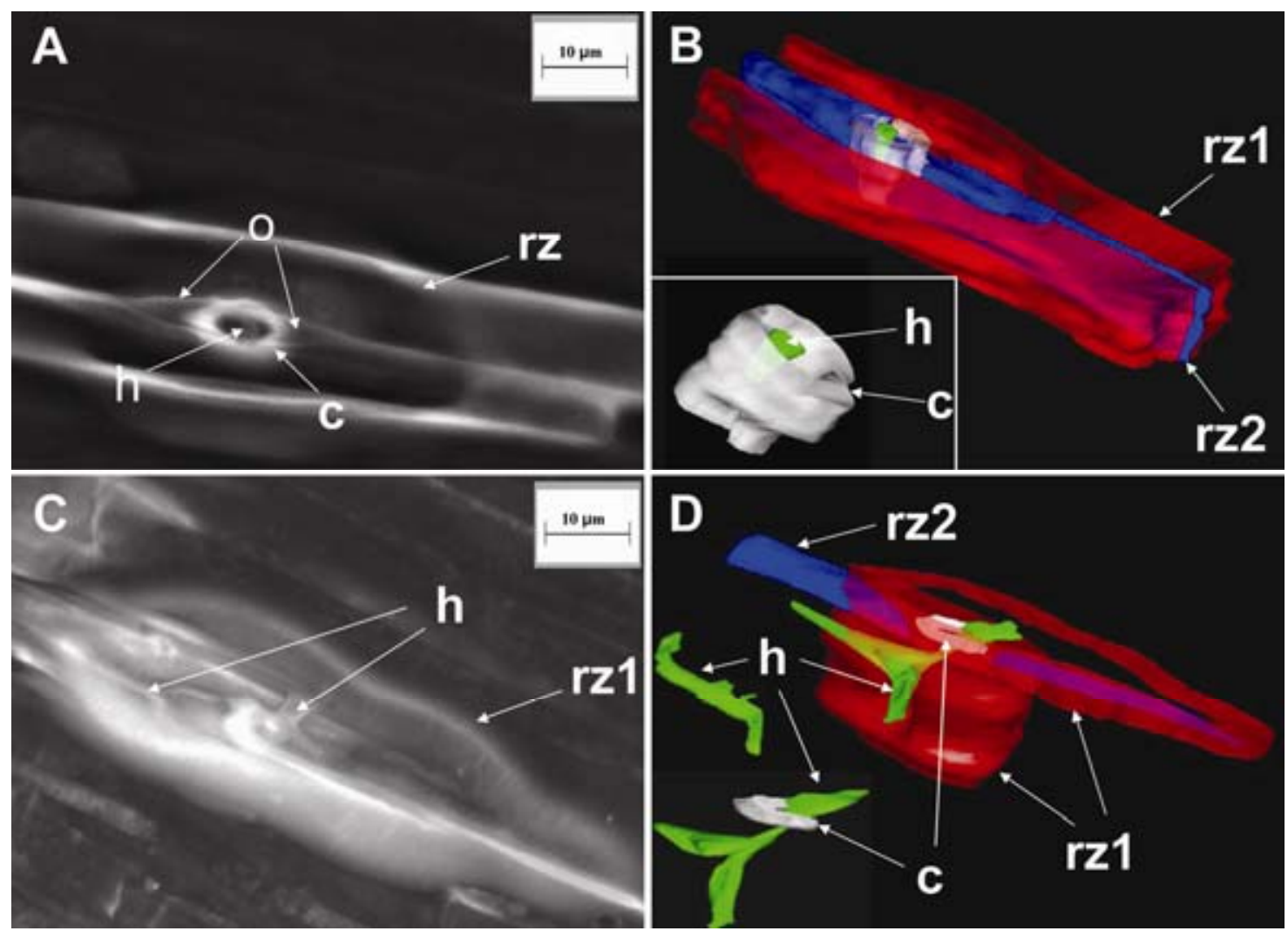

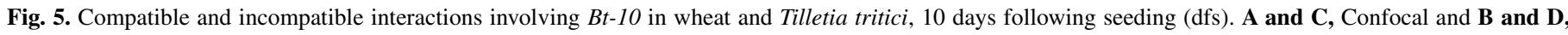

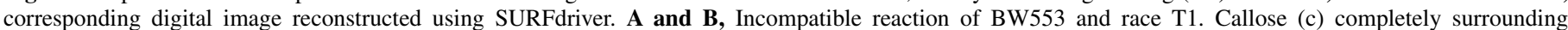

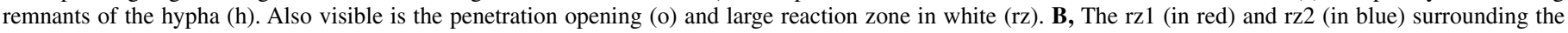

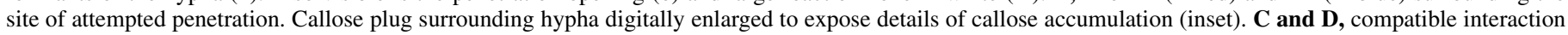

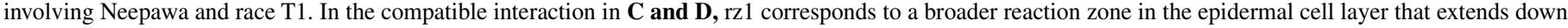

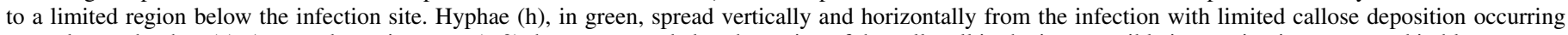
around some hyphae (c). A second reaction zone (rz2) that corresponded to the region of the cell wall in the incompatible interaction is represented in blue. 
antimicrobial factors are involved in terminating or reduced pathogen growth sufficiently so that callose could accumulate around the fungal structures.

Interpretation of the chronological course of events in this study was complicated by the nonsimultaneous spore germination characteristic of this pathogen $(30,31)$. Because early spore germination events were observed from 5 to $21 \mathrm{dfs}$, preliminary penetration events occurred as late as 18 to $21 \mathrm{dfs}$ and necessitated many repetitions of this histological study. Due to the relatively high average percentage of spores that germinate in saturated soils at $12^{\circ} \mathrm{C}$ after 5 days (30), coupled by the rapid onset of defense responses in the coleoptile involving an incompatible interaction that occurred immediately following penetration, it was possible to compare the early stages of compatible and incompatible interactions.

Events leading up to penetration including formation of both primary and secondary dikaryotic hyphae and sporidia were identical in both incompatible and compatible interactions involving BW553 and Neepawa. During the process of appressorium formation, dikaryotic hyphae assumed a wide variety of shapes and sizes as they became shorter, contorted, and deeply staining. Penetration was observed exclusively at the junction between two surface cells of the coleoptile. Early, following the formation of appressoria, autofluorescence was observed in both the incompatible and compatible interactions, from slight fluorescence 5 to $7 \mathrm{dfs}$, and reaching maximum levels 8 to $9 \mathrm{dfs}$. Initially, the levels of autofluorescence were unrelated to the nature of the resistance reaction. However, in the compatible interaction, as the fungus developed into the coleoptile from the penetration point, the area of autofluorescence assumed a diffuse appearance and spread in all directions within the coleoptile. In the incompatible interaction, the cells surrounding appressoria maintained a localized fluorescence for at least $21 \mathrm{dfs}$.
The association of autofluorescence with penetration of the wheat coleoptile by the common bunt pathogen in this study permitted a rapid quantitative assessment of penetration events. The asynchronous nature of teliospore germination resulted in a maximum number of fluorescing penetration events that averaged between 35 and 40 in $1-\mathrm{cm}$ coleoptile sections after $17 \mathrm{dfs}$ and coincides with maximum infection levels observed following exposure to only 2 weeks under the same cool optimum temperature infection conditions (7). This suggested that maximum infection generally coincides with maximum occurrence of penetration events on the coleoptile under optimum (controlled) conditions. The number of fluorescent penetration sites was numerically similar in both incompatible and compatible interactions; this coupled with other histological observations suggested that resistance reactions were affected independently of the fluorescence observed with conventional fluorescence microscopy.

Confocal imaging followed by digital reconstruction delineated two reaction zones, rz1 and rz2, in both the incompatible and compatible interactions starting at $10 \mathrm{dfs}$, based on the extent and nature of the fluorescence. These reaction zones were nondistinguishable in both the compatible and incompatible interactions using conventional microscopy (i.e., level and pattern of fluorescence in Figs. $3 \mathrm{~A}$ to $\mathrm{D}$ versus $6 \mathrm{~A}$ to $\mathrm{C}$ and $7 \mathrm{~A} ; 6 \mathrm{~A}$ versus $6 \mathrm{~B}$ ) and highlighted a clear advantage of confocal microscopy where continuous imaging of various depths within tissues is possible. A clear distinction between compatible and incompatible interactions was observed in the extent of responses for both rz1 and rz2. The more extensive host response corresponding to rz1 in the incompatible reaction compared with the more limited reaction in the compatible interaction was unlikely associated with key defense responses since fungal escape from rz1 was evident in the compatible reaction. In contrast, fungal growth was contained within rz2 that formed between cells adjacent to the penetration
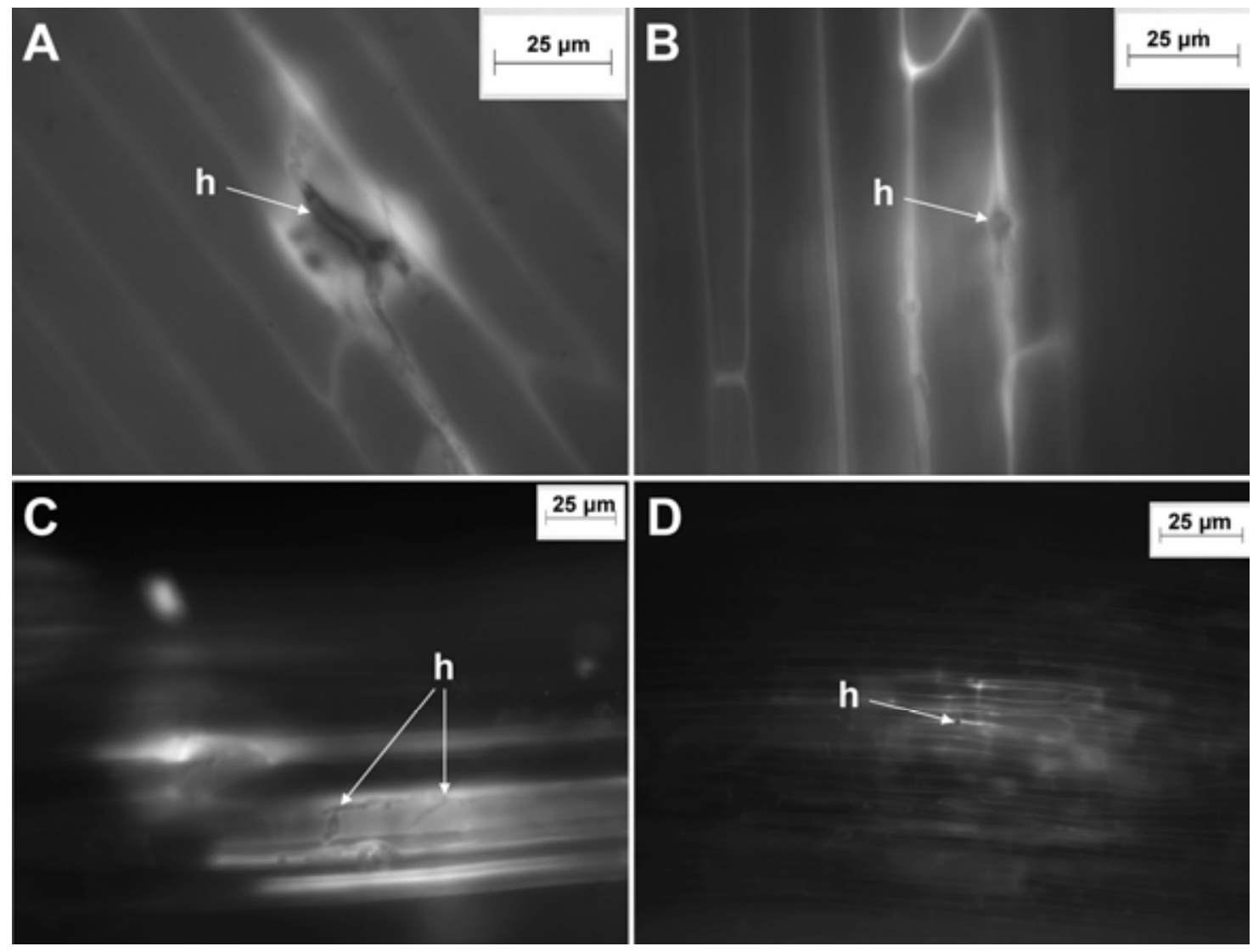

Fig. 6. Compatible interaction involving Neepawa inoculated with Tilletia tritici $\mathbf{A}$ and $\mathbf{D}$, race T27 at A, 12 days following seeding (dfs), D, 16 dfs, and $\mathbf{B}$ and $\mathbf{C}$, race T1, 15 dfs. A and B, Bright autofluorescence around hyphae (h) and between cell walls representing early penetration events; C and D, diffuse autofluorescence radiating in all directions from the infecting hypha $(\mathrm{h})$. 
site in the incompatible interaction during the early stages of infection (10 dfs); this zone was negligible in the compatible interaction. It is possible that the rz2 zone in both the incompatible interactions corresponds to the zone where critical defense responses occur that leads to the attenuation of pathogen growth. Many genes encoding PR-proteins possess signal peptides that direct their extracellular export (37) and transport, and intercellular localization could correspond with the rz2 zone. In a previous study, we demonstrated that the expression of $P R-1.1$, chitinase 2, and a lipase were delayed and expressed at lower levels in a compatible interaction involving race T1 and Neepawa compared with the rapid, higher expression levels in the incompatible interaction involving Bt-10 in BW553 (25). Since a hypersensitive response in the incompatible interaction involving $\mathrm{R}$-gene mediated resistance has not been reported for the wheatbunt pathosystem, these and other PR-proteins and defense related compounds may be key to stopping fungal ingress. In contrast, rz1 could represent nonspecific autofluorescence observed during early infection stages and correspond to a limited defense reaction in a nonhost reaction (20) whereby pathogen associated molecular patterns are recognized by the wheat plant but initial plant defense responses are somehow neutralized or silenced by the pathogen to initial defense responses. This is plausible since the early fluorescence reactions 6 to $8 \mathrm{dfs}$ appeared identical in both the incompatible and compatible interactions.
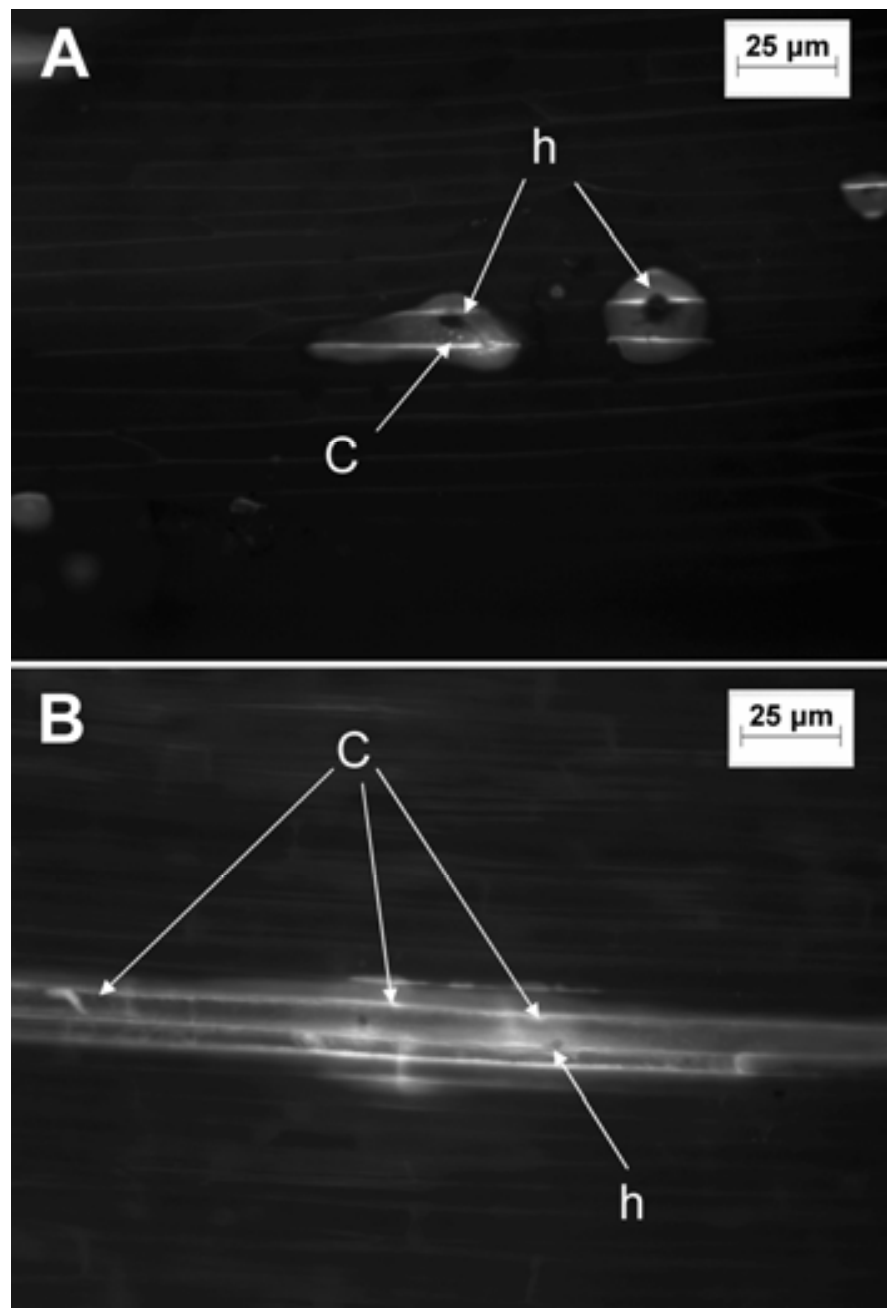

Fig. 7. Compatible interaction involving race T27 of Tilletia tritici in BW553. A, Image showing early stage of infection at 12 days following seeding (dfs). Callose (c) accumulation in vicinity of the infection hyphae (h) at the penetration site; B, later stage of infection at $13 \mathrm{dfs}$. Deposits of callose associated with cell walls proximal to the fluorescence associated with the infection site radiate in all directions from the infection hyphae.
Autofluorescence is widely regarded as an indication of expression of plant defense responses, especially as a result of the production of cell wall defense-related phenolics through the activity of peroxidases $(22,32,33)$. Our results are consistent with those reported by Park (29), who observed that emergence of the penetration peg following appressorium formation by Magnaporthe grisea is a prerequisite for triggering autofluorescence-related localized plant defense responses in rice. Autofluorescence of host cells has been associated with accumulation of phenolic defense compounds in roots of soybean mutants resistant to Phytophthora sojae (22). However, based on our observations, it appears that neither the initial autofluorescence observed during penetration nor the diffuse autofluorescence observed during fungus spread were associated with key defense responses. A similar lack of specificity of autofluorescence has been associated with necrotic lesion development in oat mutants expressing a hypersensitive response involving the crown rust pathogen, Puccinia coronata f. sp. avenae (40). Thus among cereals, autofluorescence is not necessarily a manifestation of an effective resistance response.

Rapid callose accumulation, coalescing around the infection hyphae in the epidermal cell layers of the coleoptile was the predominant microscopic symptom of incompatible reactions involving $B t-10$ and $T$. tritici race $\mathrm{T} 1$ in wheat, 8 to $20 \mathrm{dfs}$ at $12^{\circ} \mathrm{C}$. Callose was deposited in layers over the infection hyphae and on the interior cell wall of cells directly adjacent to the infection hyphae and coincided with the apparent death of the fungus. Callose synthase transcripts accumulated more rapidly, as early as $11 \mathrm{dfs}$, and at higher levels in the resistant BW553 compared with the susceptible Neepawa. In the compatible interaction with T1 and T27 in Neepawa, small deposits of callose accumulated intercellularly in apparent association with the spreading hyphae. However, callose also accumulated at high levels in the compatible interaction involving BW553 and T27, in apparent association with the developing fungus. Callose synthase transcripts were similar or higher in the compatible T27/BW553 interaction compared with that in the incompatible T1/BW553 interaction. This is likely a reflection of the ongoing callose production in response to continuing fungal spread in compatible interaction rather than being associated with fungal arrest in the incompatible interaction. Collectively, these results suggest that callose accumulation was not directly responsible for the fungistatic and eventual fungitoxic effects observed on the infection hyphae during the incompatible reaction involving Bt-10. Callose is synthesized in the plasma membrane by the enzyme callose synthase and deposited between the plasma membrane and the cell wall (23).

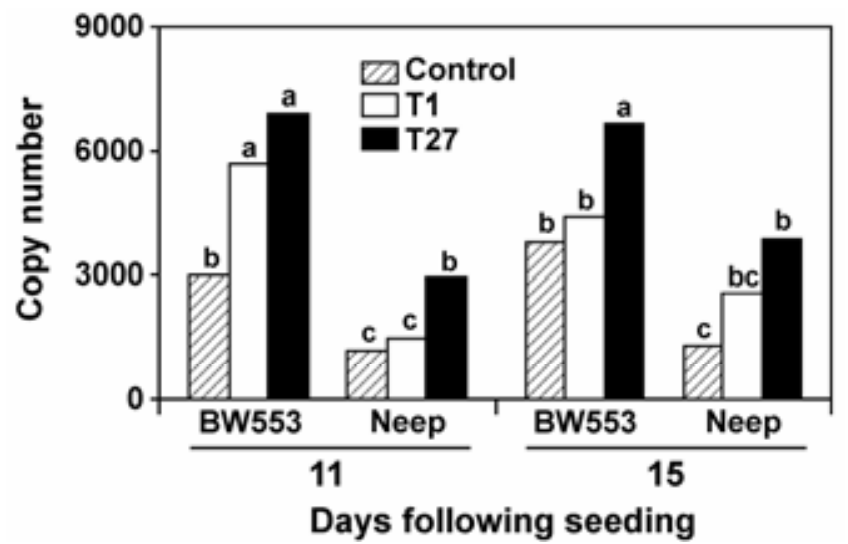

Fig. 8. Expression of wheat callose synthase transcripts in the wheat lines BW553 and Neepawa. Treatments inoculated with race T1, T27, or noninoculated (control) 11 and 15 days following seeding (dfs). Treatments within 11 and $15 \mathrm{dfs}$ followed by the same letter are not significantly different using Fisher's least significant difference test $(P<0.05)$. Copy numbers represent the number of callose synthase transcripts per $50 \mathrm{ng}$ of total RNA. 
Following membrane disruption, calcium is immediately released from the enzyme cellulose synthase (4), thereby activating the callose synthase that employs calcium as its cofactor $(12,35)$. While callose frequently accumulates in response to mechanical damage or pathogen infection, and its appearance frequently coincides with the expression of the resistance reaction, a fungistatic or fungitoxic role for callose has not been conclusively demonstrated (27). Hamiduzzaman et al. (13) demonstrated that callose inhibition led to an increase in susceptibility of grape to the downy mildew pathogen Plasmopara viticola. Conversely, callose deficient Arabidopsis mutants exhibited enhanced resistance by recruiting SA defense pathways $(18,28)$ therefore suggesting that callose may function as a suppressor of SA-dependent defense. Callose has also been proposed to protect invading pathogens by masking their presence in the plant (18). Thus, our results are consistent with the emerging view that callose is not responsible for the fungistatic and/or fungitoxic effect observed in incompatible plant-pathogen interactions (28). However, these results do not preclude the possibility that wheat callose may be fungistatic or fungitoxic to $T$. tritici but fails to accumulate sufficiently early and at high levels during compatible interactions to block pathogen ingress, as was apparent in the incompatible interaction. Because callose is known to fluoresce following staining with aniline blue, and is a feature in both compatible and incompatible interactions, it is likely that some of the nonspecific early autofluorescence associated with both compatible and incompatible interactions and the diffuse spreading autofluorescence observed in compatible responses is associated with callose deposited to varying extents in association with pathogen penetration and growth in the plant. This study illustrates the importance of utilizing more than one race per Bt-gene combination when studying resistance mechanisms since utilizing only $\mathrm{T} 1$ inoculated to BW553 and Neepawa could have resulted in an erroneous conclusion that callose was a key factor in inhibiting infection in an incompatible interaction involving Bt-10. These results will add to the current debate on the role of callose in plant defense responses.

A frequently overlooked but important consideration that impacts this study and possibly other interactions involving systemic smuts is the fact that low levels of infection are possible in incompatible interactions involving R-genes. In this study and others $(16,25)$, low levels of infection, up to $8 \%$, are observed in the incompatible interactions in lines containing R-genes under both controlled environment and field conditions. A successful infection essentially involves a competition between the pathogen and the host whereby this and some other smut fungi grow through the coleoptile, a series of embryonic leaves, and finally gain establishment in the embryonic growing point prior to upward extension of the developing spike $(34,39)$. Therefore, it is unlikely that defense responses initiated in an incompatible interaction are maintained much beyond the point of penetration or that a systemic resistance response is involved in resistance to this type of pathogen. Otherwise, the pathogen could never maintain the growth rate necessary to attain even low levels of infection and proliferate in the developing spike, if it was subjected to a continuous defense response. The failure of defense-related responses to stop the pathogen in $100 \%$ of the infection attempts in an incompatible interaction may be due to local environmental effects that are known to suppress resistance expression (7). Previously, we demonstrated that the stage of seedling development can also affect the expression of some PR-proteins induced by JA and SA (26), and for some PR-proteins, responsiveness to induction by these signaling compounds decreased with plant age. Additionally, tillers initiated later were most likely to become infected (26). Therefore, it is possible that resistance responses following very late infection are less successful in curbing pathogen development. It is likely that the resistance response in the Bt10 by race $\mathrm{T} 1$ interaction in BW553 wheat remains restricted to the localized region surrounding the penetration site. How environmental variation affects expression of PR- and other defenserelated proteins induced by common bunt is unknown. Low temperature conditions necessary for infection $(7,15)$ can have a marked effect on the expression of PR-proteins (9).

Our study also confirmed that many of the time-course details of infection of Bt-10 with T1 were similar to those detailed by Woolman (39) for the varieties Hussar and Martin, which possess the $B t-1$ and $B t-7$ resistance genes, respectively (16). A similar sequence of events has been observed for an incompatible interaction involving $U$. hordei on barley (17). Thus, at an anatomical level, race-specific gene-for-gene interactions for cereals and some seedling infecting smuts share common morphological features of defense responses. Conversely, infection studies of winter wheat by $T$. controversa Kühn in Rabenh, the dwarf bunt pathogen that is very closely related to $T$. tritici and that shares a gene-for-gene interaction with the same common bunt Bt-genes (16), have shown that this fungus progresses inwards from the coleoptile into the second leaf primordium, but fails to establish itself in the main growing point in an R-gene mediated incompatible interaction (5). These authors also failed to report any callose or gel accumulation in an incompatible interaction suggesting that incompatible responses to dwarf bunt may therefore be substantially different than those involving common bunt in wheat possessing similar race-specific Bt-genes. These differences may be attributed to the fact that the authors employed a different Bt-gene, likely $B t-8$, or different mounting and staining procedures (5). The difference in defense expression may also be due to the entirely different environmental conditions that prevail during infection of dwarf bunt that consist of up to 4 to 16 weeks incubation at near zero temperatures $(16,31)$. Under these low temperature conditions, some defense responses may be inhibited while others may be induced $(7,9)$.

In summary, we demonstrated that host perception of pathogen invasion in both compatible and incompatible interactions, based on autofluorescence in epidermal cells adjacent to the penetrating hyphae, occurs as early as 5 to $6 \mathrm{dfs}$ and the rapid accumulation of callose around invading fungal hyphae during the infection in incompatible host interactions is a predominant morphological feature in the resistance reaction. However, based on morphological evidence and expression of callose synthase in both compatible and incompatible interactions, it was apparent that callose accumulation was part of the defense response but was unlikely responsible for the termination of infection.

\section{ACKNOWLEDGMENTS}

We thank T. Despins for her able technical assistance and G. Bakkeren for his critical reading of this manuscript. The partial funding from the Agriculture and Agri-Food Canada Matching Investment Initiative against the Canadian Wheat Board Check-off Fund is acknowledged.

\section{LITERATURE CITED}

1. Cherewick, W. J. 1953. Smut diseases of cultivated plants in Canada. Canada Department of Agriculture, Ottawa.

2. Churchward, J. G. 1940. The initiation of infection by bunt of wheat (Tilletia caries). Ann. Appl. Biol. 27:58-66.

3. Dangl, J. L., and Jones, J. D. G. 2001. Plant pathogens and integrated defense response to infection. Nature 411:826-833.

4. Doblin, M. S., Kurek, I., Jacob-Wilk, D., and Delmer, D. P. 2002. Cellulose biosynthesis in plants: From genes to rosettes. Plant Cell Physiol. 43:1407-1420.

5. Fernandez, J. A., Duran, R., and Schafer, J. F. 1978. Histological aspects of dwarf bunt resistance in wheat. Phytopathology 68:1417-1421.

6. Gaudet, D. A., and Puchalski, B. L. 1989. Races of common bunt (Tilletia caries and T. foetida) in western Canada. Can. J. Plant Pathol. 60:415-418.

7. Gaudet, D. A., and Puchalski, B. L. 1995. Influence of temperature on the expression of bunt resistance genes to races of common bunt (Tilletia tritici and T. laevis) in spring wheat differentials. Can. J. Plant Sci. 75:745-749. 
8. Gaudet, D. A., Puchalski, B. L., Kozub, G. C., and Schaalje, G. B. 1993. Susceptibility and resistance in Canadian spring wheat cultivars to common bunt (Tilletia tritici and T. laevis). Can. J. Plant Sci. 73:12171224.

9. Gaudet, D. A., Laroche, A., Frick, M., Davoren, J., Puchalski, B., and Ergon, A. 2000. Expression of plant defense-related (PR-protein) transcripts during hardening and dehardening of winter wheat. Physiol. Mol. Plant Pathol. 57:15-24

10. Goates, B. J. 1996. Common bunt and dwarf bunt. Pages 12-25 in: Bunt and Smut Disease of Wheat: Concepts and Methods of Disease Management. R. D. Wilcoxson and E. E. Saari, eds. CIMMYT, D.F. Mexico.

11. Goates, B. J., and Hoffmann, J. A. 1979. Somatic nuclear division in Tilletia species pathogenic on wheat. Phytopathology 69:592-598.

12. Gressel, J., Michaeli, D., Kampel, V., Amsellem, Z., and Warshawsky, A. 2002. Ultralow calcium requirements of fungi facilitate use of calcium regulating agents to suppress host calcium-dependent defenses, synergizing infection by a mycoherbicide. J. Agric. Food Chem. 50:63536360.

13. Hamiduzzaman, M. M., Jakab, G., Barnavon, L., Neuhaus, J.-M., and Mauch-Mani, B. 2005. $\beta$-Aminobutyric acid-induced resistance against downy mildew in grapevine acts through the potentiation of callose formation and jasmonic acid signaling. Mol. Plant-Microbe Interact. 18:819-829.

14. Hansen, F. 1958. Anatomische Untersuchungen über Eindringen und Ausbreitung von Tilletia-arten in Getreidepflanzen in Abhängigkeit vom Entwicklungszustand der Wirtpflanze. Phytopathol. Z. 34:169-208.

15. Hoffmann, J. A. 1982. Bunt of wheat. Plant Dis. 66:979-986.

16. Hoffmann, J. A., and Metzger, R. J. 1976. Current status of virulence genes and pathogenic races of the wheat bunt fungi in the Northwestern USA. Phytopathology 66:657-666.

17. Hu, G. G., Linning, R., and Bakkeren, G. 2003. Ultrastructural comparison of a compatible and incompatible interaction triggered by the presence of an avirulence gene during early infection of the smut fungus, Ustilago hordei, in barley. Physiol. Mol. Plant Pathol. 62:155-166.

18. Jacobs, A. K., Lipka, V., Burton, R. A., Panstruga, R., Strizhov, N., Schulze-Lefert, P., and Fincher, G. B. 2003. An Arabidopsis callose synthase, GSL5, is required for wound and papillary callose formation. Plant Cell 15:2503-2513

19. Johansen, D. A. 1940. Plant Microtechnique. McGraw-Hill Book Co., New York.

20. Jones, J. D. G., and Dangl, J. L. 2006. The plant immune system. Nature 444:323-329.

21. Keen, N. T. 2002. A century of plant pathology: A retrospective view on understanding host-parasite interactions. Annu. Rev. Phytopathol. 38:3148.

22. Kosslak, R. M., Chamberlin, M. A., Palmer, R. G., and Bowen, B. A. 1997. Programmed cell death in the root cortex of soybean root necrosis mutants. Plant J. 11:729-745

23. Kudlicka, K., and Brown, R. M., Jr. 1997. Cellulose and callose biosynthesis in higher plants. I. Solubilization and separation of $(1 \rightarrow 3)$ and $(1 \rightarrow 4)-\beta$-glucan synthase activities from mung bean. Plant Physiol. 115:643-656.

24. Lowther, C. V. 1950. Chlamydospore germination in physiologic races of Tilletia caries and Tilletia foetida. Phytopathology 40:590-603.
25. Lu, Z.-X., Gaudet, D. A., Frick, M., Puchalski, B., Genswein, B., and Laroche, A. 2005. Identification and characterization of genes differentially expressed in the resistance reaction in wheat infected with Tilletia tritici, the common bunt pathogen. J. Biochem. Mol. Biol. 38:420431.

26. Lu, Z.-X., Gaudet, D. A., Puchalski, B., Despins, T., Frick, M., and Laroche, A. 2006. Inducers of resistance reduce common bunt infection in wheat seedlings while differentially regulating defense-gene expression. Physiol. Mol. Plant Pathol. 67:138-148.

27. Maor, R., and Shirasu, K. 2006. The arms race continues: Battle strategies between plants and fungal pathogens. Curr. Opin. Microbiol. 8:399-404.

28. Nishimura, M. T., Stein, M., Hou, B.-H., Vogel, J. P., Edwards, H., and Somerville, S. C. 2003. Loss of a callose synthase results in salicylic aciddependant disease resistance. Science 301:969-972.

29. Park, G., Xue, C., Zheng, L., Lam, S., and Xu, J. R. 2002. MST12 regulates infectious growth but not appressorium formation in the rice blast fungus Magnaporthe grisea. Mol. Plant-Microbe Interact. 15:182192.

30. Purdy, L. H., and Kendrick, E. L. 1957. Influence of environmental factors on the development of wheat bunt in the Pacific Northwest. I. Effect of soil moisture and soil temperature on spore germination. Phytopathology 47:591-594.

31. Russell, B. W., and Mills, D. 1994. Morphological, physiological, and genetic evidence in support of a conspecific status for Tilletia caries, $T$. controversa, and T. foetida. Phytopathology 84:576-582.

32. Sherf, B., Bajar, A. M., and Kolattukudy, P. E. 1993. Abolition of an inducible highly anionic peroxidase activity in transgenic tomato. Plant Physiol. 101:201-206.

33. Smith, M. M., and O'Brian, T. P. 1976. Distribution of autofluorescence and esterase and peroxidase activities in the epidermis of wheat roots. Aust. J. Plant Physiol. 6:201-209.

34. Swinburne, T. R. 1963. Infection of wheat by Tilletia caries (DC.) TUL., the causal organism of bunt. Trans. Br. Mycol. Soc. 46:145-156.

35. Thonat, C., Boyer, N., Penel, C., Courduroux, J. C., and Gaspar, T. 1993. Cytological indication of the involvement of calcium and calcium-related proteins in the early responses of Bryonia dioica to mechanical stimulus. Protoplasma 176:133-137.

36. Tottman, D. R., and Makepeace, R. J. 1979. An explanation of the decimal code for the growth stages of cereals with illustrations. Ann. Applied Biol. 93:221-234.

37. van Loon, L. C., and van Strien, E. A. 1999. The families of pathogenesisrelated proteins, their activities, and comparative analysis of PR-1 type proteins. Physiol. Mol. Plant Pathol. 55:85-97.

38. Veronese, P., Ruiz, M. T., Coca, M. A., Hernandez-Lopez, A., Lee, H., Ibeas, J. I., Damsz, B., Pardo, J. M., Hasegawa, P. M., Bressan, R. A., and Narasimhan, M. L. 2003. In defense against pathogens. Both plant sentinels and foot soldiers need to know the enemy. Plant Physiol. 131:1580-1590.

39. Woolman, H. M. 1930. Infection phenomena and host reactions caused by Tilletia tritici in susceptible and nonsusceptible varieties of wheat. Phytopathology 20:637-652.

40. Yu, G. X., Braun, E., and Wise, R. P. 2001. Rds and Rih mediate hypersensitive cell death independent of gene-for-gene resistance to the oat crown rust pathogen Puccinia coronata f. sp. avenae. Mol. PlantMicrobe Interact. 14:1376-1383. 